\title{
Comportamiento a la corrosión atmosférica marina de aceros autopatinables con estructura ferrítico perlítica y ferrítico martensítica
}

\author{
Marine atmospheric corrosion behavior of \\ structural ferritic pearlitic and ferritic \\ martensitic weathering steel
}

\author{
Alfredo Artigas ${ }^{1}$, Oscar Bustos1, Konstantin Sipos ${ }^{1}$, \\ Nelson F. Garza-Montes-de-Oca ${ }^{2}$, Alberto Monsalve ${ }^{1}$, \\ Juan Mena ${ }^{1}$, Rodrigo Seco ${ }^{1}$
}

\author{
${ }^{1}$ Departamento de Ingeniería Metalúrgica, Facultad de Ingeniería, Universidad de Santiago de Chile, Av. L. B. O`Higgins \\ 3363, Estación Central, Santiago, Chile. CP 9160000. \\ email: alfreo.artigas@usach.cl; oscar.bustos@usach.cl; ksiposh@yahoo.com; rodrigo.seco@usach.cl; \\ alberto.monsalve@usach.cl; juan.mena@usach.cl \\ ${ }^{2}$ Facultad de Ingeniería Mecánica y Eléctrica, Universidad Autónoma de Nuevo León, San Nicolás de los Garza, Nuevo \\ León, México C.P 66450. \\ email: nelson.garza@gmail.com
}

\section{RESUMEN}

La industria del acero se encuentra constantemente enfrentada al desafío del desarrollo de nuevos y mejores aceros para diversas necesidades, tales como la necesidad de aceros estructurales con mayor resistencia a la corrosión marina que los que existen en la actualidad, y que además posean una mayor resistencia mecánica. Por ello y en pos de lo anteriormente expuesto es evidente que, investigar en forma sistemática tanto la influencia de los elementos aleantes, como las variables del proceso de fabricación de aceros estructurales resistentes a la corrosión marina es relevante para el desarrollo del mercado acerero nacional.

El presente trabajo tendrá por objetivo, la comparación de comportamiento a la corrosión atmosférica marina (espesor corroído en el tiempo) entre aceros de composición autopatinable (ASTM A242 y ASTM A588) con estructuras ferrítico-perlítica y ferrítico-martensítica. Para lo anterior se realizaron ciclos de humectación salina y secado. La humectación salina se realizó en cámara de niebla salina, de acuerdo a los requerimientos presentados en la norma ASTM B177, y el secado, en una mufla a $40{ }^{\circ} \mathrm{C}$. Tras el retiro de muestras, se realizaron decapados químicos, según lo requerido en ASTM G1, para la determinación de espesor corroído.

Palabras clave: ambiente marino, corrosión acelerada, aceros autopatinables.

\section{ABSTRACT}

The steel industry constantly faces the challenge of developing new and better steels for diverse needs, on of this necessities is the use of structural steels with higher corrosion resistance to marine atmospheres and improved mechanical properties than those that currently exist. Therefore, it is necessary to systematically research both the influence in the corrosion resistant to marine atmospheres of the alloying elements, and others variables such as changes in the manufacturing process of this materials.

This work aims to compare the behavior on marine atmospheric corrosion between weathering steels (ASTM A242 and ASTM A588) with ferritic-pearlitic and ferritic-martensitic structures. Wetting and drying cycles were performed. Saline wetting was performed in a salt spray apparatus, according to ASTM B177, while 
drying was performed in a furnace at $40^{\circ} \mathrm{C}$. At the end of each cycles, the corrosion products were removed in accordance to ASTM G1.

Keywords: marine environment, accelerated corrosion, weathering steels.

\section{INTRODUCCIÓN}

El material metálico más utilizado a nivel industrial en el mundo es el acero[1], pues a un bajo precio, este combina resistencia mecánica y la posibilidad de ser trabajado mediante variedad de métodos, lo que ofrece un amplio abanico de posibilidades para la manufacturación de piezas. En función de requerimientos específicos, sus propiedades pueden ser modificadas mediante diferentes procesos de conformado, tratamientos térmicos y/o modificaciones en la composición química.

Sin embargo, la gran mayoría de los aceros sufren una degradación de sus propiedades en el tiempo debido a la corrosión ambiental. En general los óxidos superficiales se forman en capas discontinuas que se desprenden fácilmente, llevando paulatinamente a una pérdida de material que finalmente se traduce en la necesidad de reemplazar las piezas afectadas. Según un estudio de los costos asociados a esta pérdida de sus características, realizado en el año 2001 en los Estados Unidos[2], dirigido por CC Technologies para la administración Federal de Autopistas (FHWA), se estima que las pérdidas económicas del país debido a problemas relacionados con la corrosión del acero fluctúan en el orden del 5\% del producto bruto interno nacional (PIB), cifras cercanas a los 276 mil millones de dólares estadounidenses.

Es por ello que ya a principios del siglo pasado se iniciaron una serie de estudios, que tratan acerca de la influencia de elementos aleantes que modifican las propiedades del óxido superficial convirtiéndolo en una capa continua, densa y adherente, la cual retarda el avance de la corrosión. La capa de óxido formada, se denomina pátina, por lo cual estos aceros se denominan "autopatinables". La motivación principal del presente trabajo surge de la discrepancia que existe entre autores $[\underline{3}, \underline{4}, \underline{5}, \underline{6}]$ en cuanto a la resistencia a la corrosión atmosférica de aceros dual-phase al ser comparados con sus pares ferrítico-perlíticas, además otros autores $[\underline{7}, \underline{8}, \underline{9}]$ no hacen referencia al no especificar cual podría tener mejor comportamiento frente a la corrosión.

\subsection{Aceros autopatinables}

Los aceros autopatinables corresponden a la familia de aceros estructurales de Alta Resistencia y Baja Aleación (HSLA) con adición de elementos aleantes tales como $\mathrm{Cu}, \mathrm{Cr}$ y $\mathrm{Ni}$. Estos aceros son utilizados en aplicaciones estructurales tales como edificios y puentes, tanto carreteros como de ferrocarril, y en equipos rodantes como vagones ferroviarios. Debido a su mayor resistencia mecánica, es posible fabricar piezas de espesores menores, lo que se traduce en menor peso y disminución de costos.

Mientras los aceros al carbono desarrollan durante el proceso de corrosión capas de óxidos que se desprenden con facilidad, los aceros autopatinables forman una capa densa y adherente de óxido sobre su superficie que impide el avance de la corrosión. Esta propiedad se debe a la adición de elementos aleantes, principalmente Cobre y Cromo. La oxidación en comparación a los aceros al carbono ocurre de manera similar, pero la diferencia radica en que al cabo de 3 años[10] la pátina protectora se estabiliza, formando un óxido superficial denso y adherente de color oscuro, que limita seriamente el avance de la corrosión.

Para la formación de esta capa superficial, se deben tener ciertas consideraciones ya que sólo se generará con ciclos alternados secos y húmedos, y siempre que no exista polución atmosférica ácida o salina.

\subsection{Pátina Protectora}

La formación de la pátina protectora ocurre de manera natural. La corrosión superficial del acero, en presencia de la humedad, genera oxihidróxidos de hierro del tipo $\mathrm{FeOOH}$ por vía electroquímica, y su desarrollo depende de factores tales como los ciclos alternados de humectación y secado a los cuales se somete el material debido a las variaciones climáticas. Los oxihidróxidos $\mathrm{FeOOH}$ existen en varias formas alotrópicas, cuyas estructuras cristalográficas ilustran la Figura 1. En la Lepidocrocita $(\gamma-\mathrm{FeOOH})$ y en la Goethita $(\alpha-\mathrm{FeOOH}), 1 \mathrm{a})$ y $1 \mathrm{~b})$ respectivamente. 


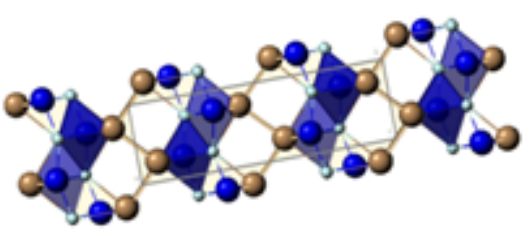

3: Lepidocrosita, $\mathrm{y}-\mathrm{FeOOH}$

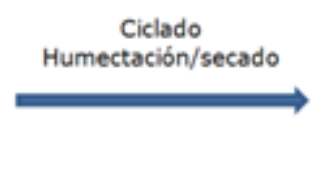

3: Goethita, a-FeOOH

Figura 1: Formas alotrópicas de los oxihidróxidos de hierro[11]: (a) Lepidocrocita y (b) Goethita.

El primer oxihidróxido que se forma corresponde a la Lepidocrocita, el cual después de transcurridos algunos ciclos de humectación y secado se transforma en una fase más estable, denominada Goethita. Las diferencias entre sus estructuras, cuya unidad básica son los octaedros de $\mathrm{Fe}(\mathrm{O}, \mathrm{OH})_{6}$ es que mientras la Lepidocrocita es una serie de estructuras octaédricas planas unidas entre sí sólo por átomos de hidrógeno, la Goethita presenta una estructura de octaedros unidos entre sí de forma más compacta, al unir los planos estructurales por octaedros y no sólo por átomos de hidrógeno. La Goethita es un oxihidróxido formado a baja velocidad de corrosión, y que madura en una estructura con menor número de defectos, ofreciendo mayor resistencia a la corrosión debido a que posee una estructura más compacta y resistente impidiendo la permeabilidad de oxígeno y agua. Es así que, para lograr potenciar la transformación de Lepidocrocita a Goethita estos aceros deben usarse sin pintura ni otro recubrimiento adicional.

Para obtener las mejores características de esta pátina protectora es necesario que el tamaño de los granos de la Goethita sean lo más pequeños posible, lo que se logra mediante la adición de elementos aleantes. Los pequeños granos de la Goethita, permiten una mayor cohesión entre granos, disminuyendo el área entre los espacios cristalinos, actuando como una barrera efectiva a los efectos de la corrosión.

La presencia de iones Cloruro en los ambientes marinos forman un estado alotrópico diferente, llamado Akaganeita $(\beta-\mathrm{FeOOH})$, que forma, alrededor de los iones Cloruro, estructuras octaédricas con un túnel central. Para evitar el efecto de los iones Cloruro, se agregan elementos aleantes tales como Níquel, Molibdeno y Titanio, que restringen la permeabilidad de las estructuras mediante transmisión selectiva de iones. Además el Níquel contemplado en la composición química del acero, permite superar eventuales problemas de fragilidad en caliente que pudieran surgir debido a la presencia de Cobre.

\subsection{Aceros dual phase}

Los aceros de alta resistencia y baja aleación, proveen un material con mejores propiedades mecánicas, al ser comparados con los aceros ordinarios. Estas mejores propiedades mecánicas no son obtenidas fácilmente, requiriendo por ejemplo, procesos de deformación en frío. Las cada vez más exigentes normativas para componentes estructurales o las requeridas en la industria automotriz, promueven la utilización de estos materiales.

En respuesta a esta necesidad se ha demostrado que aceros de baja aleación, que típicamente contienen Manganeso y Silicio, pueden exhibir tanto altas resistencias como buenas prestaciones para ser conformados si son tratados térmicamente con la finalidad de producir una matriz ferrítica con islas de martensita (10 $20 \%$ en volumen). Estos aceros han sido referidos como aceros dual phase de baja aleación (DPLA). Los aceros DPLA presentan fluencia continua, sin el típico fenómeno de fluencia heterogénea de sus pares ferrítico-perlíticos, a relativamente bajos esfuerzos (300-350MPa), lo que para la industria automotriz, por mencionar un caso, es de gran utilidad, al no desarrollar bandas de Lüders[12] durante los procesos de conformado. Los aceros más simples dentro de esta categoría, contienen $0,08-0,20 \% \mathrm{C}, 0,50-1,50 \% \mathrm{Mn}$. No 
obstante, también son adecuados aceros microaleados con Vanadio, o que eventualmente contengan pequeñas adiciones de $\mathrm{Cr}(0,50 \%)$ y $\mathrm{Mo}(0,20-0,40 \%)$.

El camino más simple para la obtención de esta estructura dúplex, se realiza mediante un recocido intercrítico, en el campo bifásico $(\alpha-\gamma)$ entre Ac1 y Ac3, típicamente a $790{ }^{\circ} \mathrm{C}$ durante varios minutos, con la finalidad de obtener pequeñas regiones austeníticas en una matríz ferrítica. Dado que es importante la transformación de estas regiones austeníticas el enfriamiento posterior ha de ser rápido o bien la austenita formada ha de tener una alta templabilidad [13]. Lo anterior puede ser logrado por ejemplo al agregar entre 0,20 y $0,40 \%$ de Mo para el caso de aceros que contienen 1,5\% de Manganeso, de esta manera, la estructura requerida puede ser obtenida por un enfriamiento al aire luego del recocido.

Si se quiere eliminar el tratamiento térmico anterior, la estructura requerida se puede obtener durante el enfriamiento luego de la laminación en caliente. Estos aceros típicamente contienen adiciones de $0,05 \% \mathrm{Cr}$ y 0,40\% Mo. Una vez completada la laminación en caliente, cerca de los $870{ }^{\circ} \mathrm{C}$, el acero forma aproximadamente $80 \%$ de ferrita al ser enfriado con agua a la salida del laminador. El enrollado de la bobina a los $510-620^{\circ} \mathrm{C}$, y su posterior enfriamiento produce la transformación de las regiones austeníticas en martensita.

\section{MATERIALES Y MÉTODOS}

Con la finalidad de evaluar la resistencia a la corrosión atmosférica marina de aceros autopatinables con estructuras ferrítico-perlíticas y ferrítico-martensíticas, en primer término, se fabricaron a escala de laboratorio, aceros ASTM A242[14] y ASTM A588[15], según el procedimiento ilustrado en la figura 2a.

Luego de la laminación en caliente se obtienen planchas de $5 \mathrm{~mm}$ de espesor. En la sección de tratamientos térmicos, se realizó una autenitización y posterior enfriamiento lento (en arena) a las planchas, para la obtención de estructuras ferrítico-perlíticas, y para el caso de las estructuras ferrítico-martensíticas, se optó por la realización de un calentamiento intercrítico a $750^{\circ} \mathrm{C}$, de acuerdo a simulaciones realizadas con el programa Thermo-Calc, considerando las composiciones químicas de los aceros fabricados, planteando como objetivo la presencia de $20 \%$ de martensita.

a)

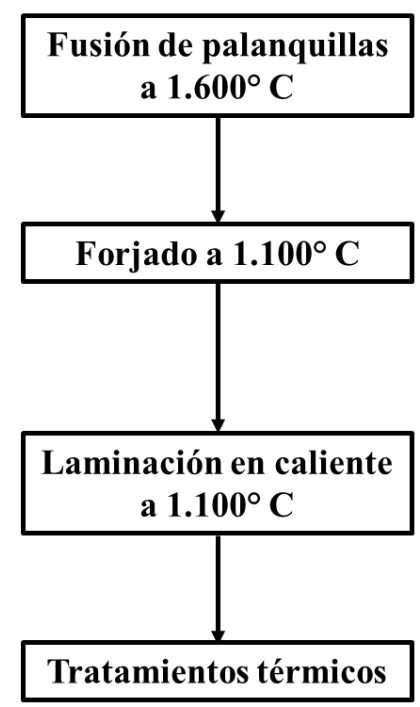

b)

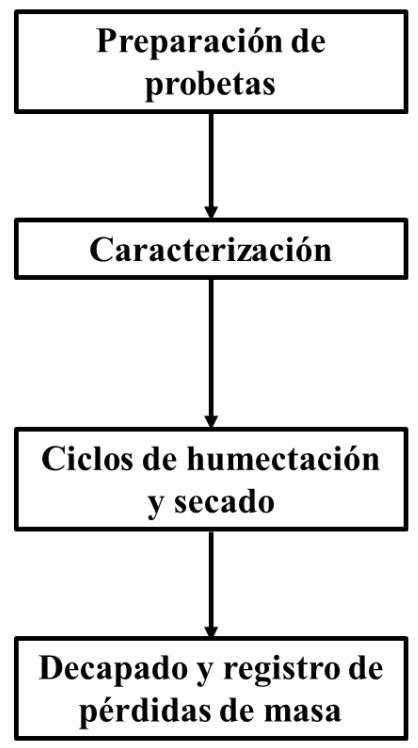

Figura 2: Esquema de procedimiento experimental.

Ya obtenidos los aceros con las estructuras de interés se procedió en conformidad a lo ilustrado en la figura $2 \mathrm{~b}$. En lo que respecta a la preparación de probetas se obtuvieron cupones de 40,00 x 30,00 x 5,00 mm, los que fueron sometidos a ciclos de humectación salina y secado, según lo presentado en la Figura 3[16]. Se 
debe destacar el lavado de las muestras realizado en cada ciclo, ya que permite la remoción de $\mathrm{NaCl}$ en exceso. La eliminación del exceso de sal permite la formación de los oxihidróxidos protectores en la superficie del acero, lo que lo provee de las características autopatinables del mismo, lo cual quedo demostrado en investigaciones anteriores del grupo de trabajo[17]. Aproximadamente cada 5 ciclos se retiraron cupones para ser sometidos a decapado en ácido clorhídrico 33\%, en conformidad a lo establecido en ASTM G1[18], lo anterior con la finalidad de evaluar la pérdida en masa de acero en función del tiempo de corrosión para todos los aceros obtenidos.

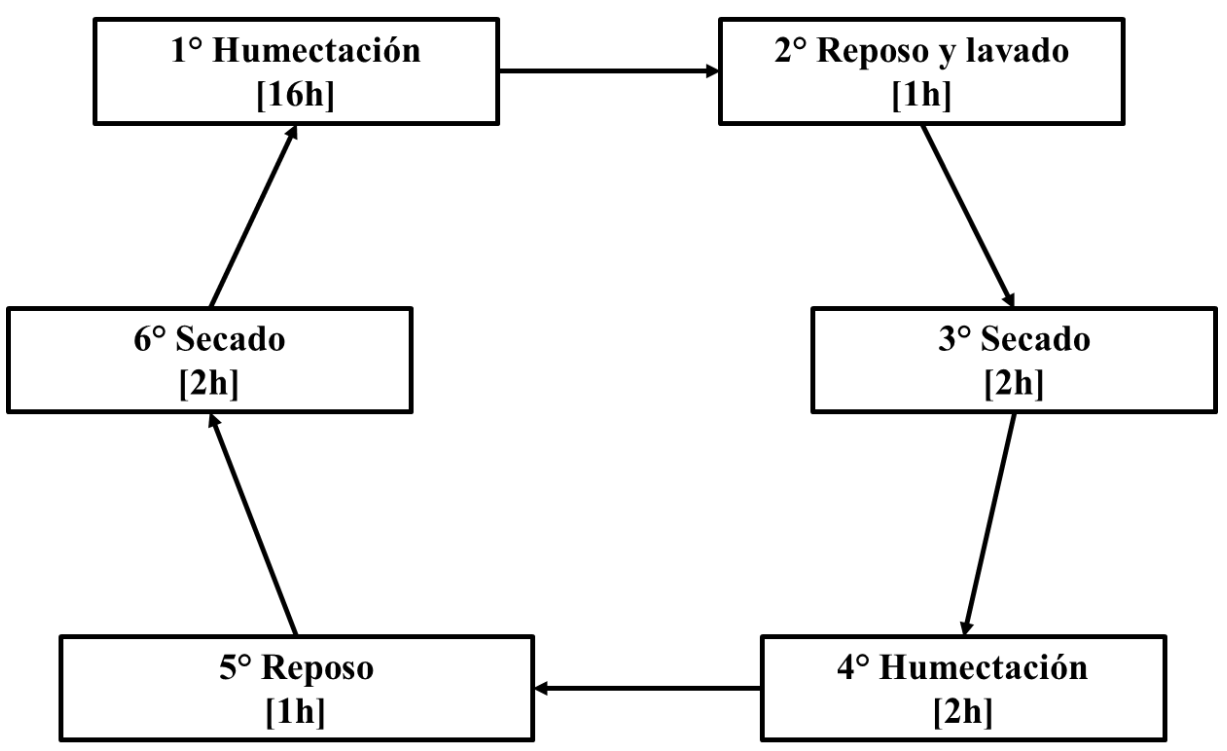

Figura 3: Representación de un ciclo de humectación salina y secado.

\section{RESULTADOS}

\subsection{Análisis de composición química}

Los resultados de los análisis químicos, obtenidos por espectrometría de emisión óptica, de los distintos aceros fabricados, en muestras obtenidas de las palanquillas, se ilustran en la Tabla 1.

Tabla 1: Resultados de análisis químico.

\begin{tabular}{c|c|c|c|c|c|c}
\hline Muestra & \%C & \% Mn & \% Cr & \% Ni & \% Cu & \% Fe \\
\hline A242 fabricado & 0,102 & 0,350 & 0,830 & 0,333 & 0,315 & Resto \\
\hline Referencia A242 & 0,110 & 0,310 & 0,820 & 0,310 & 0,300 & Resto \\
\hline A588 fabricado & 0,186 & 0,800 & 0,383 & 0,282 & 0,317 & Resto \\
\hline Referencia A588 & 0,130 & 1,030 & 0,560 & 0,015 & 0,33 & Resto \\
\hline
\end{tabular}

\subsection{Evaluación de propiedades mecánicas}

Los resultados de propiedades mecánicas para los aceros fabricados, obtenidas luego de laminación y tratamientos térmicos se presentan en la Tabla 2 
Tabla 2: Propiedades mecánicas.

\begin{tabular}{c|c|c|c}
\hline Muestra & $\begin{array}{c}\text { Esfuerzo de } \\
\text { fluencia } \\
\text { MPa }\end{array}$ & $\begin{array}{c}\text { Esfuerzo máximo } \\
\text { MPa }\end{array}$ & $\begin{array}{c}\text { Elongación } \\
\%\end{array}$ \\
\hline A242 F-P & 369 & 516 & 30 \\
\hline A242 F-M & 445 & 681 & 21 \\
\hline A588 F-P & 387 & 520 & 26 \\
\hline A588 F-M & 530 & 749 & 17 \\
\hline
\end{tabular}

\subsection{Análisis metalográfico}

La Figura 4 presenta imágenes de los aceros estudiados en sus estructuras ferrítico-perlíticas (F-P) y ferríticomartensíticas (F-M), luego de ser preparadas para análisis metalográfico, en conformidad a ASTM E3[19], y luego de ser atacadas con Nital 3\% durante 5 segundos.
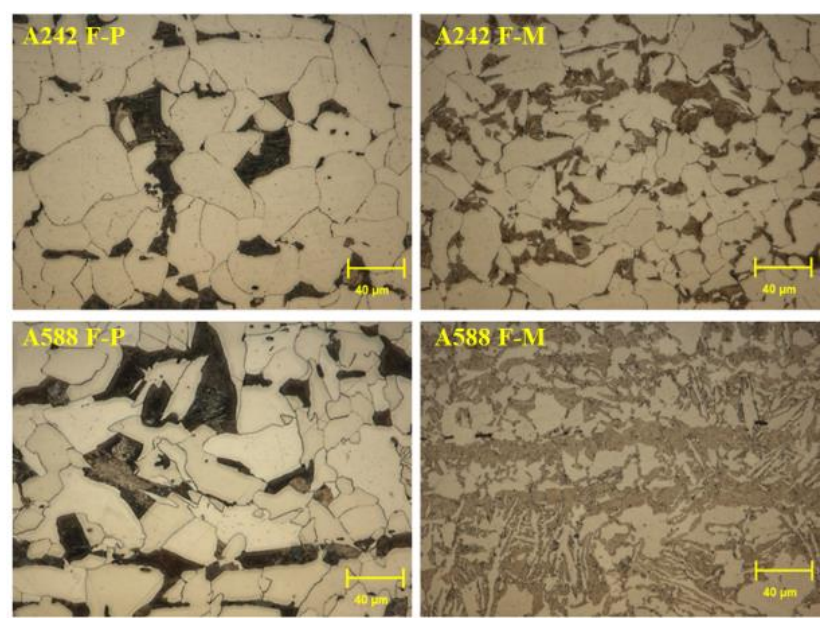

Figura 4: Metalografías obtenidas para los cuatro aceros estudiados.

De la Figura 4 se observa que los tratamientos térmicos realizados para la obtención de las estructuras de interés fueron adecuados.

\subsection{Resultados de corrosión acelerada}

Como se mencionó, aproximadamente cada 5 ciclos de humectación salina y secado, se retiraron muestras para la determinación del espesor corroído en función del tiempo previo decapado según ASTM G1. Con los anteriores resultados se obtuvieron curvas de penetración de corrosión $(\mu \mathrm{m})$ vs número de ciclos (Figura 5), y velocidad de corrosión (mpy) versus número de ciclos, Figura 6. 


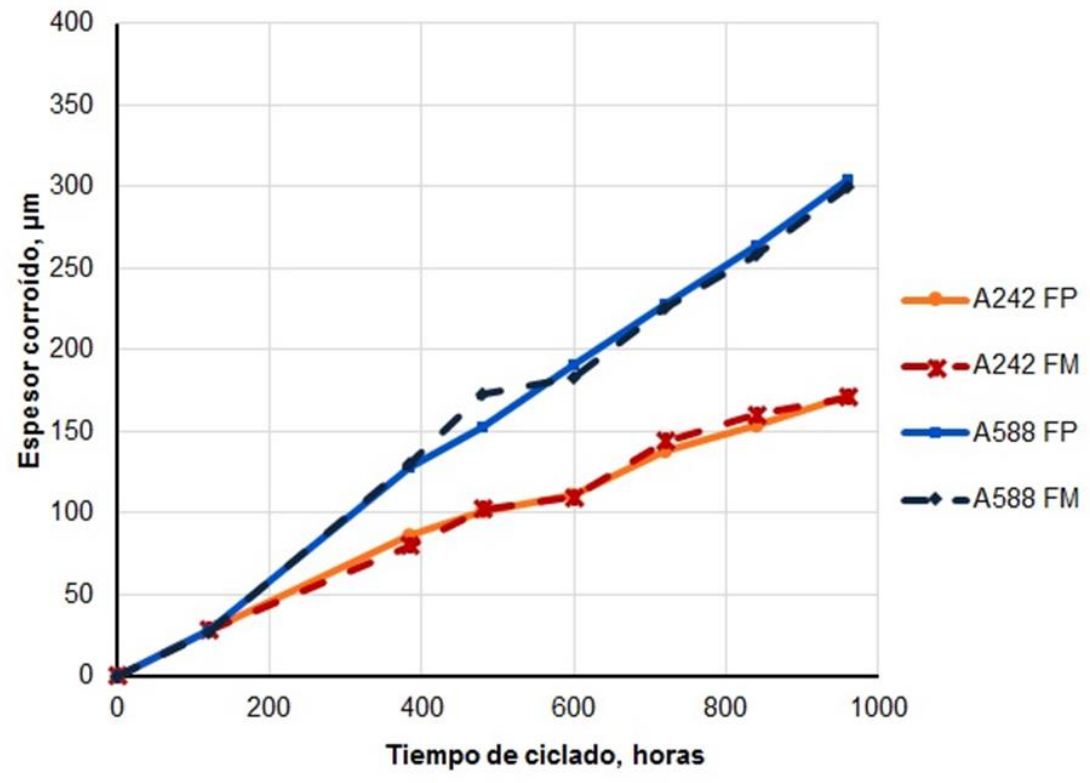

Figura 5: Espesor corroído versus número de ciclos.

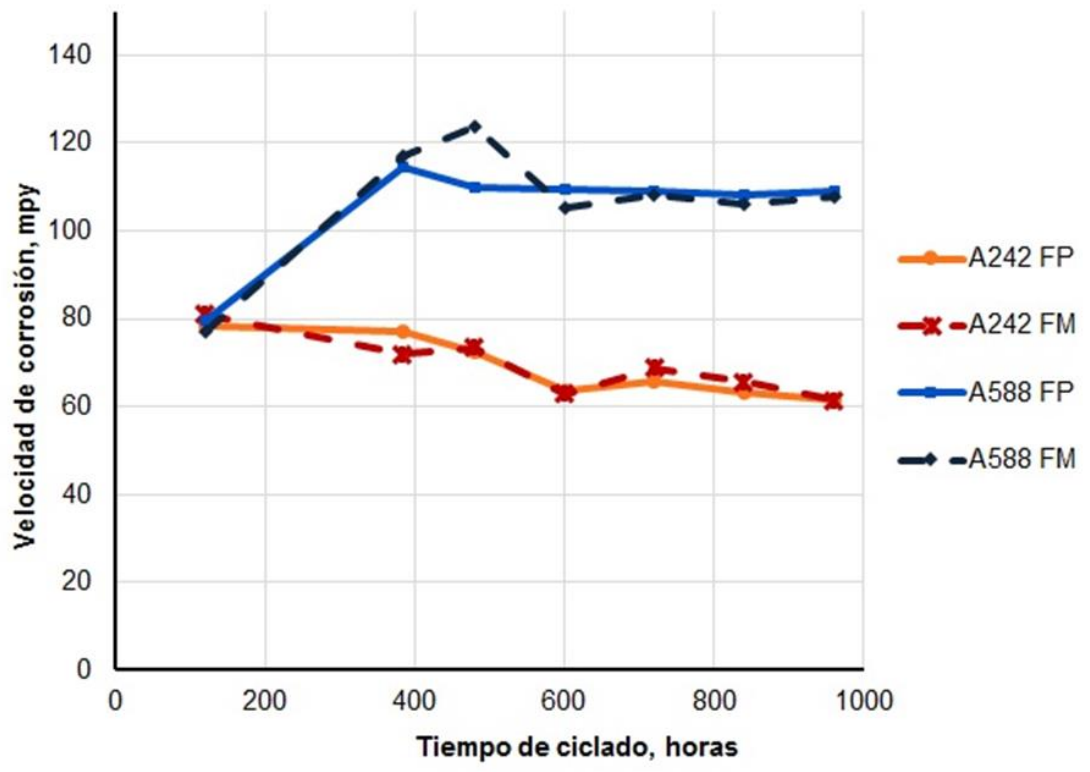

Figura 6: Velocidad de corrosión versus número de ciclos.

\section{DISCUCIONES}

De las Figuras 5 y 6 se infiere que, tanto para estructuras ferrítico-perlíticas como ferrítico-martensíticas, el acero de composición concordante con ASTM A242 presenta una mayor resistencia a la corrosión atmosférica salina (representada por espesores corroídos menores en todos los ciclos) que el que presenta una composición química concordante con un acero ASTM A588, justificado en la mayor presencia de elementos aleantes tales como Cromo y Níquel, lo que es concordante con resultados de ensayos realizados en tiempo 
real, presentados en la norma ASTM G101. Análogo es el resultado observado para las velocidades de corrosión.

Para el caso de las velocidades de corrosión se observa que en ambos aceros la velocidad de corrosión disminuye luego del ciclo 15, ya que en esta etapa se forman las capas de óxidos y oxihidróxidos iniciales. Una vez formada la Lepidocrocita, ésta se transforma en Goethita, protegiendo al acero contra el avance de la oxidación. Este efecto valida que el ensayo de corrosión acelerada con ciclos de humectación salina y secado planteado para aceros patinables del tipo de los ensayados produce una disminución y estabilización de velocidad de corrosión y por tanto permite replicar las condiciones de intemperie bajo las cuales de desarrolla la pátina protectora.

Se ha observado que la resistencia a la corrosión atmosférica marina de aceros con estructuras ferrítico-perlíticas y ferrítico-martensíticas dependería fundamentalmente de la composición química, lo que permitiría obtener aceros más resistentes, luego de la aplicación de un tratamiento térmico sin afectar su resistencia a la corrosión atmosférica marina. Allam et al. [4], han planteado que existe diferencia en su resistencia a la corrosión, principalmente en los primeros años, sin embargo otros autores[ $\underline{3}, \underline{5}, \underline{6}, \underline{7}]$, no se plantean esta inquietud, es por ello que esta inquietud queda resulta, al menos en los aceros estudiados, es decir el tipo de estructura, no afecta de manera importante su resistencia al corrosión atmosférica marina de estos aceros.

\section{CONCLUSIONES}

El uso de aceros ASTM A588 y ASTM A242, con microestructura dual-phase es interesante en condiciones de corrosión atmosférica, puesto que sus mejores características mecánicas comparadas con los de microestructura ferrítico-perlíticos se obtienen sin deterioramiento de la resistencia a la corrosión atmosférica, cuya afirmación, hasta ahora, no estaba bien definida [ $\underline{3}, \underline{4}]$.

\section{AGRADECIMIENTOS}

Los autores desean agradecer a Conicyt Proyecto Fondecyt $N^{\circ} 1120537$ y a Dicyt de la Universidad de Santiago de Chile, por el apoyo al presente trabajo.

\section{BIBLIOGRAFÍA}

[1] STEINER, R., "ASM Metals Handbook Volume 01 Properties and Selection Irons, Steels, and HighPerformance Alloys", 10 ed, Ohio, ASM International, pp 233.

[2] GERHARDUS K., MICHIEL H., NEIL T., "Corrosion Costs and Preventive Strategies in the United States", Cost of Corrosion Study Unveiled, n. FHWA-RD-01-156, 2002.

[3] ZHANG, C., CAI, D., ZHANG, E., et al., "Effect of Dual-Phase Treatment on the Corrosion Behavior of Weathering Steel 09CuPCrNi", Advanced Materials Research, v.740, pp. 586-590, 2013.

[4] ALLAM, T., ABBAS, M. "Mechanical Properties, Formability, and Corrosion Behavior of Dual Phase Weathering Steels Developed by an Inter-Critical Annealing Treatment", Steel Research International, v.83, pp. 231-240, 2015.

[5] ISHIKAWA, T., KUMAGAI, M., YUSUKAWA, A. "Influences of metal ions on the formation of $\gamma$ FeOOH and magnetite rusts", Corrosion Science, v. 44, pp. 1073-1086, 2002.

[6] YAMASHINA, M, UCHIDA, H. "Recent Research and Development in Solving Atmospheric Corrosion Problems of Steel Industries in Japan", Hyperfine Interactions, v. 139-140, pp. 153-166, 2002.

[7] ZHANG, C., LIAO, B., CAI, D., et al., "Development of hot-rolled dual-phase weathering steel Cu-PCr-Ni-Mo", Materials \& Design, v. 28, pp. 1760-1766, 2007.

[8] ZHANG, C., CAI, D., ZHAO, T., et al., "Simulation of hot-rolled dual-phase weathering steel 09CuPCrNi”, Material Characterization, v. 55, pp. 378-382, 2005.

[9] ZHANG, C., CAI, D., LIAO, B, "Using direct hot-rolling approach to obtain dual-phase weathering steel $\mathrm{Cu}-\mathrm{P}-\mathrm{Cr}-\mathrm{Ni}-\mathrm{Mo}$ ”, Journal of Materials Science, v. 45, pp. 490-495, 2010.

[10] JARAMILLO, B., CALDERÓN, J., et al., "Evaluación electroquímica de aceros autoprotectores en condiciones simuladas de laboratorio", Revista Facultad de Ingeniería, n. 37, pp. 200-210, Jul. 2006 
[11] KIMURA M., SUZUKI, T., "Fe(O,OH $)_{6}$ Network Structure of Rust Formed on Weathering Steel”, Surface and Interface Analysis, v. 35, pp. 66-71, Jan. 2003.

[12] GONZALES, J.L., Metalurgia Mecánica, 1 ed, México, Editorial Limusa S.A., 2003.

[13] ASTM Standard A255, 2003, "Standard Test Methods for Determining Hardenability of Steel", ASTM International, West Conshohocken, PA, DOI: 10.1520/A0255-10R14, www.astm.org.

[14] ASTM Standard A242, 2013 "Standard Specification for High-Strength Low-Alloy Structural Steel", ASTM International, West Conshohocken, PA, DOI: 10.1520/A0242_A0242M, www.astm.org.

[15] ASTM Standard A588, 2010, "Standard Specification for High-Strength Low-Alloy Structural Steel, up to $50 \mathrm{ksi}$ [345 MPa] Minimum Yield Point, with Atmospheric Corrosion Resistance", ASTM International, West Conshohocken, PA, DOI: 10.1520/A0588_A0588M-10, www.astm.org.

[16] ARTIGAS, A., BUSTOS, O., SIPOS, K., et al., "Implementación de un ensayo de corrosión acelerada para la evaluación de penetración de corrosión en aceros resistentes a la corrosión atmosférica", In: 13 ${ }^{\mathrm{a}}$ Congreso nacional en Ciencias y Tecnología de Materiales, Puerto Iguazú, Argentina, pp. 20-21, Agosto 2013.

[17]ARTIGAS, A., MONSALVE, A., SIPOS, K., et al., "Development of accelerated wet-dry cycle corrosion test in marine environment for weathering steels", Corrosion Engineering, Science and Technology DOI: 10.1179/1743278215Y.0000000007, 2015.

[18] ASTM Standard G1, 2011, "Standard Practice for Preparing, Cleaning, and Evaluating Corrosion Test Specimens", ASTM International, West Conshohocken, PA, DOI: 10.1520/G0001-03R11, www.astm.org.

[19] ASTM Standard E3-11, 2011, "Standard Guide for Preparation of Metallographic Specimens", ASTM International, West Conshohocken, PA, DOI: 10.1520/E0003-11, www.astm.org. 